\title{
A NOTE ON THE PAPER "ON COMPLETENESS IN METRIC SPACES AND FIXED POINT THEOREMS"
}

\author{
Deepak Khantwal ${ }^{1}$ and Umesh Cuandra Gairola ${ }^{2 *}$ \\ ${ }^{I}$ Department of Mathematics, Graphic Era Hill University, Dehradun, Uttarakhand, India \\ ${ }^{2}$ Department of Mathematics, H.N. B. Garhwal University, Campus Pauri (Garhwal), Uttarakhand, India
}

*Corresponding Author Email id: ucgairola@rediffmail.com

Received: 16.11.2020; Revised: 5.12.2020; Accepted: 9.12.2020

(C)Society for Himalayan Action Research and Development

\begin{abstract}
In the present note, we show that the assumption of continuity used in the fixed point theorem of Gregori et al. (Results Math. 73 (2018), no. 4, Art. 142, 13) can be relaxed to some weaker version of continuity. More precisely, we prove a fixed point theorem for orbitally continuous and k-continuous mappings in weak G-complete metric space and provide an appropriate example to show that our result is not only valid for continuous mappings but also for some discontinuous mappings. Moreover, we apply our main result to establish a common fixed point theorem for two self-mappings.
\end{abstract}

Subject Classification: Primary 47H10; Secondary 54H25

Keywords: Fixed Point, orbitally continuous maps, k-continuous maps, metric space.

\section{Introduction}

Grabiec (1988) introduced the notion of the GCauchy sequence, which is a weaker form of Cauchy sequence in the setting of the fuzzy metric space. He used this concept to determine the classical Banach contraction principle for fuzzy metric spaces. Generally, a compact metric space is complete in the classical sense, but unfortunately, a compact fuzzy metric space fails to be complete in Grabiec sense. To overcome this disadvantage, Gregori et al. (2018) introduced the concept of weak G-completeness and adopted this concept to generalize Grabiec's

Banach Contraction Principle. The class of weak G-complete metric space lies between the class of completeness and compactness, and the structure of weak G-completeness forms a bridge between the compactness and completeness. The study of such intermediate classes has become an active area of research in analysis over the years (Atsuji 1958; Beer 1986).
Recently Gregori et al. (2018) characterized weak $\mathrm{G}$-completeness using nested sequences of nonempty closed sets in the classical metric space and shown that the concepts of completeness, Gcompleteness, and weak $\mathrm{G}$-completeness agree in ultra-metric spaces. They employed the concept of weak G- completeness to establish a general fixed point theorem for a continuous self-mapping $f$. Also, they showed that the Boyd and Wong's fixed point theorem (Boyd and Wong 1969) can be stated for weak G-complete spaces by weakening the condition on the gauge function.

In this note, we prove a fixed point theorem for orbitally continuous and k-continuous mapping in weak G-complete metric space, and show that the assumption of continuity used in Theorem 1.7 of Gregori et al. (2018) can be relaxed further to some weaker notions of continuity. We also have an appropriate example to show that our result is not only valid for continuous mappings but also 
for some discontinuous mappings. As an application of our main result, we establish a common fixed point for two self-mappings in weak G-complete metric space.

In this paper, we accept the following definitions, which are essential in this sequel and contained in (Ciric 1974; Gregori et al. 2018; Pant and Pant 2017).

Definition1.1. (Gregori et al. 2018). Let $(X, d)$ be a metric space. A sequence $\left(x_{n}\right)$ such in $X$ is said to be G-Cauchy if $\lim _{n \rightarrow \infty} d\left(x_{n}, x_{n+1}\right)=0$ and $X$ is called G-complete if every G- Cauchy sequence in $X$ is convergent.

Definition1.2. (Gregori et al. 2018). Let $(X, d)$ be a metric space. A sequence $\left(x_{n}\right)$ such in $X$ is called G-convergent if it is G-Cauchy and it has, atleast a cluster point, and $X$ is called weak GConvergent if every G-Cauchy sequence in $\mathrm{X}$ is G-convergent.

We recall the set

$o(x ; f)=\left\{f^{n} x: n=0,1,2, \ldots\right\}$ is called the orbit of the self-mapping $f$ at the point $x \in X$.

Definition 1.3. (Ciric 1974; Dhage 1985; Dhage 2017). A self-mapping $f$ of a metric space $(X, d)$ is said to be orbitally continuous at a point $z \in X$ if for any sequence $\left(x_{n}\right) \subset O(x ; f)$ for some $x \in X,\left(x_{n}\right) \rightarrow z$ implies $\left(f x_{n}\right) \rightarrow f z$ as $n \rightarrow \infty$.

It is important to note that every continuous selfmapping is orbitally continuous in a complete metric space, but the converse need not be true. The following example shows that the orbitally continuity of function $f$ does not implies continuity of function $f$.

Example 1.4. Suppose $X=[0,2]$ be a usual metric space endowed with usual metric $d$. Define a self-mapping $f$ on $X$ by

$$
f(x)=\left\{\begin{array}{l}
1, \text { if } x \in[0,1), \\
0, \text { if } x \in[1,2] .
\end{array}\right.
$$

Then it is easy to see that the function $f$ is not continuous at point $x=1$ but orbitally continuous therein. To see this, let $\left(x_{n}\right) \subset O(x ; f)$ such that $\left(x_{n}\right) \rightarrow 1$ then $\left(f x_{n}\right) \rightarrow 0 \neq f(1)$ as $n \rightarrow \infty$.

Definition 1.5. (Pant and Pant 2017). A selfmapping $f$ of a metric space $(X, d)$ is called $k$-continuous, $\quad k=1,2,3, \ldots \quad$ if $f^{k} x_{n} \rightarrow f z$, whenever $\left(x_{n}\right)$ is a sequence in $X$ such that $f^{k-1} x_{n} \rightarrow z$ in $X$.

It is obvious by definition of $k$-continuity that every continuous mapping $f$ of a metric space $(X, d)$ is $k$-continuous mapping and continuity coincides with the notion of 1-continuity. However, k-continuity of a function (for $k \geq 2$ ) does not implies the continuity of function. The following example illustrates this fact.

Example 1.6. Let $X=[0,6]$ and $d$ be a usual metric space $X$. Define a mapping $f: X \rightarrow X$ by $f(x)=\left\{\begin{array}{l}3, \text { when } x \in[0,3], \\ 0, \text { when } x \in(3,6]\end{array}\right.$

then it is easy to verify that the function $f$ is not continuous at point $x=3$. But the function $f^{2} x=3$, for all $x$ in $[0,6]$, is continuous or the function $f$ is 2-continuous, for all values of $x$ in $X$.

The following result is proved in Gregori et.al. (2018)

Theorem 1.7. Let $(X, d)$ be weak G-complete metric space and let $f: X \rightarrow X$ be continuous mapping. Suppose there exists $x \in X$ such that the iterative sequence $\left(x_{n}\right)$ is G-Cauchy, where $x_{1}=f(x) ; x_{n}=f\left(x_{n-1}\right)$ for $n=2,3,4, \ldots$. Then $f$ has a fixed point (more precisely, the cluster points of $\left(x_{n}\right)$ are fixed points for $\left.f\right)$. If in addition $\quad d(f x, f y)<d(x, y), \quad$ for $x \neq y, x, y \in X$, then the fixed point is unique.

\section{Main result}

Now we state our main result. 
Theorem 2.1. Let $(X, d)$ be a weak G-complete metric space and $f: X \rightarrow X$ be a $k$-continuous or orbitally continuous mapping. Suppose there exists $x_{0} \in X$ such that the iterative sequence $\left(x_{n}\right)$ is G-Cauchy,

Where $\quad x_{1}=f\left(x_{0}\right) ; x_{n}=f\left(x_{n-1}\right)$ for $n=2,3,4, \ldots$ then $f$ has a fixed point. If in addition $d(f x, f y)<d(x, y)$ for $\neq y, x, y \in X$, then the fixed point is unique.

Proof: Let us suppose that there exists $x_{0}$ in $X$ such that the sequence $\left(x_{n}\right)$ where $x_{1}=f\left(x_{0}\right)$ and $x_{n}=f\left(x_{n-1}\right)$ for $n=2,3, \ldots$ is G-Cauchy sequence. Then $\left(x_{n}\right)$ has at least one cluster point (say), $c$, thus there exists a subsequence $\left(x_{p_{n}}\right)$ of $\left(x_{n}\right)$ such that $\left(x_{p_{n}}\right)$ converges to $c$. Now following the proof of Theorem 1.7, we may prove that $\left(x_{p_{n}-1}\right)$ is also converges to $c$.

Suppose that $f$ is k-continuous and $\left(f^{k-1}\left(x_{p_{n}-k}\right)\right)$ converges to $c$ then $k$-continuity of $f$ implies that $\left(f^{k}\left(x_{p_{n}-k}\right)\right.$ ) converges to $f(c)$. Hence from the above, we conclude that $f(c)=c$ that is, $c$ is a fixed point of function $f$.Furthermore we suppose that $f$ is orbitally continuous and $\left(x_{p_{n}-1}\right)$ converges to $c$ then orbital continuity of $f$ implies that $\left(f\left(x_{p_{n}-1}\right)\right)$ converges to $f(c)$. This yield $f(c)=c_{p}$ that is $c$ is a fixed point of $f$.

The following example illustrates our result.

Example 2.2. Let $A=[0,1]$ and $B=\{2,3,4, \ldots, n, \ldots\}, n \in N$ (Natural number). Take $X=A \cup B$ and suppose $X$ endowed with the usual metric $d$ We define a function from $f: X \rightarrow X$ such that:

$f(x)=\left\{\begin{array}{c}1 / 3, \text { if } x \in[0,1 / 2], \\ 0, \quad \text { if } x \in[1 / 2,1] \cup B .\end{array}\right.$
Then it is easy to verify that $X_{x}$ is a weak Gcomplete non-compact and non-complete metric space (see Example 1 in [8]). Consider, let $x_{0} \in X$ be an arbitrary point in $X_{x}$ then iterative sequence $x_{n}=f\left(x_{n-1}\right)=1 / 3$, for $n=1,2,3, \ldots$, and $\lim _{n \rightarrow \infty}\left|x_{n+1}-x_{n}\right|=0$. Hence $\left(x_{n}\right)$ is a GCauchy and we also observe that $d(f x, f y)<d(x, y)$ for all $x, y \in A \cup B$. Thus, all the assumptions of Theorem 2.1 are satisfied and $f$ has unique fixed point at $x=1 / 3$.

\section{Application}

In this section we prove a common fixed point theorem as an application of our main result.

Theorem 3.1. Let $(X, d)$ be a metric space and $f, g: X \rightarrow X$ both are eitherk-continuous mappings or orbitally continuous mappings such that $f(X) \subseteq g(X)$. Suppose that $g(X)$ is weak Gcomplete metric subspace of $X$.

(i). Assume that for some $x_{0} \in X$ there exists an iterative sequence $\left(a_{n}\right)$ in $g(X)$, where $\quad a_{0}=f\left(x_{1}\right)=g\left(x_{0}\right)$ and $a_{n}=f\left(x_{n+1}\right)=g\left(x_{n}\right), n=1,2,3, \ldots$ is GCauchy in $g(X)$. Then $f$ and $g$ has a coincidence point, that is $f(z)=g(z)$ for some $z \in Y$.

(ii). Further $f$ and $g$ has common fixed point, that is $f(z)=g(z)=z$, provided that $f$ and $g$ commute at coincidence point means $f g(z)=g f(z) \quad$ whenever $(z)=g(z)$.

(iii). If in addition, $d(f x, f y)<d(g x, g y)$ holds for all $x, y \in X$ and $x \neq y$ then the common fixed point is unique.

Proof: First of all, we define a function $F: g(X) \rightarrow g(X)$ such that $F(a)=f\left(g^{-1}(a)\right)$ for each $a \in g(X)$. 
Next, we show that $F$ is well define. To see this, we observe that $f(x) \subseteq g(X)$ and for all $x \in g^{-1}(a)$,

$F(a)=f(x)$.

Take $\quad x, y \in g^{-1}(a) \quad$ such that $f(x)=b, f(y)=c$ then, since $g(x)=g(y)$, we have $b=c$, so $F$ is well defined. Since assumption (i) implies that for some $x_{0} \in X$, there exists a sequence $\left(a_{n}\right)=\left(g\left(x_{n}\right)\right)$ in $g(X)$ is G-Cauchy. Then by definition of $F$, the sequence $\left(F\left(a_{n}\right)\right)$ is also G-Cauchy in $g(X)$.

Further for $a \neq b$ and

$a, b \in g(X), g^{-1}(b) \cap g^{-1}(a)=\phi$, then for $x \in g^{-1}(a)$ and $y \in g^{-1}(b)$, we have $d(f x, f y)=d(F x, F y)<d(a, b)=d(g x, g y)$

Hence all the conditions of Theorem 2.1 are satisfied for function. Therefore $F$ has a unique fixed point (say) $w$ in $(X)$. As for any $z \in g^{-1}(w), f z=F w=w=g z$, so $z$ is a coincidence point of $f$ and $g$. If $f$ and $g$ are commuting at coincidence point $z$, then $f z=g z$ implies

$f f z=f g z=g f z=g g z$ and $f w=g w$.

If $f z \neq f f z$, then

$d(w, f w)=d(f z, f f z)<d(g z, f z)=d(w, f w)$

a contraction. Hencew is a common fixed point of $f$ and $g$.

We conclude the proof by showing the unicity of the common fixed point. For this, suppose $v \neq w$ is another common fixed point of $f$ and $g$. Then from (3.1), we have

$d(w, y)=d(f w, f y)<d(g w, g y)=d(w, v)$, which is a contradiction. Hence $v=w$.

\section{Acknowledgement}

The Authors are very much indebted to the referee for his valuable suggestions towards the improvement of the manuscript.

\section{References}

Atsuji M (1958) Uniform continuity of continuous functions of metric spaces. Paci. J. Math. 8(1): 11-16.

Beer G (1986) More about metric spaces on which continuous functions are uniformly continuous. Bull. Aust. Math. Soci. 33(3): 397-406.

Boyd DW and Wong JSW (1969) On nonlinear contractions Proc. Am. Math.Soc. 20(2): 458-464.

Ciric LB (1974) On some maps with a nonunique fixed point. Publ. Inst. Math 17: 31, 52-58

Dhage, BC (1985) Some result for the maps with a nonunique fixed point. Indian J. Pure Appl. Math. 16, 245-256.

Dhage BC (2017) Basic hybrid fixed point theorems for contraction mäpplings in partially ordered metric spaces. Thai J. Math. 15, 721-732.

Grabiec M (1988) Fixed points in fuzzy metric spaces. Fuzzy set syst. 27(3): 385-389

Gregori V, Minana J, Roig B and Sapena A (2018) On completeness in metric spaces and fixed point theorems. Results Math. 73: 4, Art. 142, 13.

Gregori V, Minana JJ and Sapena A (2018) On Banach contraction principles in fuzzy metric spaces. Fixed Point Theory 19 (1), 235-247.

Pant A and Pant, RP (2017) Fixed points and continuity of contractive maps, Filomat 31(11): 3501-350.

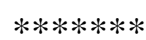

